\title{
ANÁLISIS DEL TRATAMIENTO DE LAS DESIGUALDADES Y LA RESOLUCIÓn PACÍFICA DE CONFLICTOS EN LA ORDER AUTONÓMICA REGULADORA DEL CURRÍCULO DE LA ESO ER CASTILLA Y LEÓn
}

\author{
Analysis of the Treatment of Inequalities and the \\ Peaceful Conflict Resolution in the Education \\ Regulation in Castilla y León
}

Noelia Santamaría-Cárdaba

Universidad de Valladolid

E-mail: noelia.santamaria.cardaba@uva.es

ORCID: https://orcid.org/0000-0001-6864-9330
Juan José Mediavilla Merino

Universidad de Valladolid

E-mail: juanjo.mediavilla@soc.uva.es ORCID: https://orcid.org/0000-0001-5638-4347
○ Autores

Este artículo analiza dos contenidos clave de la Educación para la Ciudadanía Global en la normativa educativa de la Educación Secundaria Obligatoria en la Comunidad Autónoma de Castilla y León: las desigualdades y la resolución pacífica de conflictos. Se utiliza como metodología el análisis documental, pues se trata del análisis de textos legislativos. Los resultados muestran que estas dimensiones se tratan en la legislación, pero de forma muy superficial, solo en tres asignaturas y sin que las cuestiones globales adquieran una presencia importante. Se concluye que la normativa de Castilla y León presenta carencias a la hora de formar ciudadanos que adquieran una perspectiva global de los problemas existentes en otras partes del mundo y sus causas.

Educación para la ciudadanía global; currículo educativo; Educación Secundaria Obligatoria; resolución pacífica de conflictos; desigualdades.

Global Citizenship Education; educational curriculum; Secondary Education; peaceful conflict resolution; inequalities. 
This article analyses two key contents of Global Citizenship Education in the educational regulations of Compulsory Secondary Education in the Autonomous Community of Castilla and León: inequalities and peaceful conflict resolution. Documentary analysis is used as a methodology, since it is the analysis of legislative texts. The results show that these dimensions are dealt with in the legislation, but in a very superficial way, only in three subjects and without global issues acquiring an important presence. It is concluded that the legislation of Castilla and Leon presents deficiencies when it comes to forming citizens who acquire a global perspective of the problems existing in other parts of the world and their causes.

\section{Introducción}

La adquisición de una competencia global es una cuestión que actualmente genera un especial interés en los debates educativos; tanto es así que desde el año 2018 el informe internacional de evaluación de estudiantes conocido como PISA ha incluido en su estudio la competencia global, la cual pretende que el alumnado sea competente a la hora de "examinar cuestiones locales, globales e interculturales, comprender y apreciar diferentes perspectivas y visiones del mundo, interactuar con éxito y de manera respetuosa con los demás y actuar de modo responsable hacia la sostenibilidad y el bienestar colectivo" (OECD, 2018, p. 5).

Incluso, la ONU (2015) incluye en su Agenda 2030 los Objetivos de Desarrollo Sostenible (ODS), para cuya consecución la ciudadanía juega un papel relevante. Esta Agenda 2030 tiene carácter global y busca promover el desarrollo socioeconómico y ambiental abarcando "por primera vez [...] cuestiones como la pobreza no extrema, la desigualdad y la inclusión social, el crecimiento "verde" y los problemas ambientales globales, y en especial el cambio climático" (Sanahuja, 2015, p. 379). Caride (2017) añade que estos 17 ODS y sus 169 metas buscan dar respuesta a los retos globales, entre los que destaca lograr una educación de calidad para todas las personas, promover el consumo responsable, la igualdad de género o "la promoción de sociedades más pacíficas, justas e inclusivas” (Caride, 2017, p. 252).

Acercar a la ciudadanía estos objetivos conlleva que desde el ámbito educativo se forme a las personas para que adquieran una formación que promueva la consecución de los ODS, siendo este uno de los principales retos a los que se enfrentan los sistemas educativos actuales (Murga-Menoyo, 2018). Por este motivo, para lograr el cumplimiento de dichos objetivos, no solo las políticas sociales, sino también las educativas deben ir encaminadas a promover que la ciudadanía impulse una sociedad más justa y, para ello, resulta innegable la importancia de la educación para el desarrollo y la ciudadanía global (EDCG).

Ahora bien, ¿qué se entiende por EDCG? Establecer una definición de este término no es una tarea sencilla debido que está sometido a múltiples connotaciones (Mayoral, 2011; Ortega, Cordón-Pedrosa y Sianes, 2013). En este sentido, se han tomado como referencia las definiciones propuestas por autores como Bourn (2015), Andreotti (2014), Pashby, Costa, Stein y Andreotti (2020) o Pashby (2015), entre otros, para establecer la noción de EDCG presente en este estudio; en este caso, se concibe la EDCG como un proceso educativo que busca promover la formación de una ciudadanía activa que piense críticamente y actúe en defensa de los derechos humanos para transformar la sociedad actual en una más sostenible, justa y 
responsable (Santamaría-Cárdaba, 2018). Ante estos propósitos resulta indispensable que en las normativas que rigen la educación formal se promueva la construcción de una ciudadanía que resuelva los conflictos de forma pacífica y que actúe en contra de las desigualdades; por esta razón, el presente estudio se centra en analizar la presencia de estas dos cuestiones en la normativa que regula el currículo de Educación Secundaria Obligatoria (en lo sucesivo, ESO).

Cabe añadir que autores como De Prada y López (2008), Lapponi (2013) o Soriano (2009) afirman que desde el ámbito educativo no solo se transmiten conocimientos, sino que también se aprende a vivir en sociedad; por ello, fomentar pautas de comportamiento que promuevan el diálogo, el consenso y la cooperación resultan imprescindibles para mejorar la convivencia y erradicar la violencia. Como indican Ortega (2006) y Soriano (2009), educar para la paz, y la resolución pacífica de conflictos, facilita que los jóvenes sean solidarios y comprendan la importancia de ser justos.

Adicionalmente, la sociedad multicultural actual hace que la escuela deba tratar las diferencias sin provocar desigualdad y también, educar desde una perspectiva integradora y respetuosa (Rebollo, 2006). En esta línea, Vila (2009) afirma que en el mundo globalizado tratar la "tolerancia se vuelve aquí casi una necesidad social, educativa y ciudadana” (p. 47), pues de esta forma se mejorará la convivencia y se promoverá la construcción de ciudadanos responsables. Por este motivo, es importante desarrollar políticas que contribuyan "a promover la equidad, la excelencia y la calidad del sistema educativo desde la cultura de la paz, haciendo especial énfasis en la superación de las desigualdades” (Alguacil, Bloqué, y Alcalde, 2019, p. 81).

Por consiguiente, y tomando como referencia lo expuesto en líneas anteriores, este estudio tiene como objetivo analizar la presencia de dos aspectos clave dentro de la Agenda 2030, como son las desigualdades y la resolución pacífica de conflictos, en la normativa que establece el currículo de ESO en Castilla y León (España).

\section{Metodología}

La metodología empleada es el análisis documental, pues se trata de una técnica adecuada para el tipo de textos que vamos a abordar, de tipo legislativo. Además, es una metodología adecuada en la sociedad del conocimiento (Salazar-Gómez y Tobón, 2018) y conforme con el objetivo propuesto en el presente estudio: averiguar cómo se tratan las desigualdades y la resolución de conflictos en el currículo que regula la etapa educativa de la ESO en Castilla y León. Al ser un único documento analizado, no se han necesitado herramientas informáticas de análisis de texto, sino que se ha hecho de forma manual; concretamente, se fueron subrayando en el documento los aspectos en los que se centraba este estudio.

El análisis documental se basa en la búsqueda de teorías y categorías de conocimiento a través de operaciones de tipo mecánico e intelectual. Es decir, se trata de extraer las nociones más importantes de un documento dado (Valles, 1999) — en nuestro caso, la legislación educativa de Castilla y León_. En este tipo de análisis se propone una corriente integral (Molina, 1989), que consistiría en una observación rigurosa, tanto interna y externa de los documentos para extraer la información necesaria para la comprensión del contenido (Bermeo-Yaffar et al., 2016). Para ello, utilizaremos preguntas de investigación y diferentes categorías de análisis aplicadas al estudio y examen de algunos conceptos clave para la EDCG. De este modo, nuestra técnica de análisis será el de la cartografía conceptual (Bermeo-Yaffar, 2016), adaptada a nuestro caso,
La adquisición de una competencia global es una cuestión que actualmente genera un especial interés en los debates educativos 
que consiste en la búsqueda y análisis de la información — previamente categorizada— para comprender cada uno de los conceptos (Salazar-Gómez y Tobón, 2018).

Para seguir esta metodología, proponemos la consideración de los siguientes elementos del texto: 1) clasificación; 2) análisis; 3) explicación y 4) conclusión. La primera de ellas queda incluida en este mismo punto de explicación metodológica y selección del texto a analizar. La segunda pertenece al cuarto epígrafe, donde analizaremos las categorías y niveles de análisis del texto. Por su parte, la tercera y la cuarta se tratan en el apartado de conclusiones.

La legislación que en la que se centra este análisis es: la ORDEN EDU/362/2015, de 4 de mayo, por la que se establece el currículo y se regula la implantación, evaluación y desarrollo de la ESO en la comunidad de Castilla y León. Se trata, por tanto, de un texto de tipo jurídico, por cuanto emana de fuentes legales y tiene validez normativa, es decir, establece normas del derecho. Como se señalaba en la introducción, y con el objetivo de guiar el análisis se han establecido dos categorías: 1) desigualdad — cuestiones ligadas con el tratamiento de la desigualdad en todas sus formas en la normativa- - 2) resolución de conflictos de forma pacífica —aspectos ligados a la resolución de conflictos-.

\section{Resultados}

A continuación, se pasan a detallar los resultados obtenidos en cada una de las categorías propuestas tras realizar el análisis a la normativa ya nombrada.

\subsection{Desigualdad}

En la primera de las categorías, la referida a la desigualdad, en la ORDEN EDU/362/2015 se aprecia una primera referencia en los contenidos actitudinales de la asignatura de Economía, aunque únicamente en una enumeración breve:

La economía juega, además, un papel central en la configuración de valores y actitudes y, por ello, adquieren especial relevancia los contenidos actitudinales relacionados con la solidaridad entre personas, grupos y pueblos; la valoración de las relaciones no competitivas; la actitud ante las desigualdades económicas; la importancia de la conservación del medio natural y el desarrollo sostenible para la calidad de vida; el rechazo ante el consumo innecesario; la igualdad efectiva entre hombres y mujeres y la igualdad de trato y la no discriminación por cualquier condición o circunstancia personal o social. (ORDEN EDU/362/2015, p. 32118)

Del mismo modo, también aparece en los contenidos del Bloque 4. Economía e ingresos y gastos del Estado, incluyendo las "desigualdades en virtud del género" y, en los criterios de evaluación, "el impacto para la sociedad de la desigualdad de la renta”, así como en los estándares de aprendizaje evaluables (ORDEN EDU/362/2015, p. 32121).

En el área de Geografía e Historia también se encuentra una referencia a la categoría de desigualdad: "Y, en el tercero, el análisis y explicación de las desigualdades en el espacio geográfico actual y sus repercusiones. Así como el negativo impacto de la acción humana sobre el medioambiente y las medidas correctoras que se derivan" (ORDEN EDU/362/2015, p. 32141).

En la asignatura Valores Éticos se localiza también referencia a la desigualdad, específicamente en los estándares de aprendizaje evaluables del tercer y cuarto curso, tanto en el conteni-

\section{La sociedad} multicultural actual hace que la escuela deba tratar las diferencias sin provocar desigualdady también, educar desde una perspectiva integradora $y$ respetuosa 
do referido a la Declaración Universal de Derechos Humanos (ORDEN EDU/362/2015, p. 32355), como en el que se ocupa del poder político, sus formas y su legitimación (ORDEN EDU/362/2015, p. 32358).

\subsection{Resolución pacífica de conflictos}

Respecto a la segunda categoría, lo primero que llama la atención es que hay varias referencias al conflicto — bien sea de manera genérica, o personal, o social— pero ninguna a la paz. Así, la primera que aparece se refiere a la resolución de conflictos dentro del aula y el clima de convivencia y se encuentra en el artículo 53, el cual trata sobre las funciones de los equipos docentes:

Establecer actuaciones para mejorar el clima de convivencia del grupo, interviniendo coordinadamente en la resolución los conflictos que puedan surgir en el seno del grupo, sin perjuicio de las competencias que correspondan a otros órganos en materia de prevención y resolución de conflictos. (ORDEN EDU/362/2015, p. 32086)

Como se puede observar, se trata de conflictos internos, no a los que se pueda referir la EDCG en un sentido global a pesar del valor de desarrollar un clima de convivencia adecuado tanto en el centro educativo, como dentro del aula. Del mismo modo, en varias asignaturas aparecen referencias al conflicto de diferentes formas. Por ejemplo, en Geografía e Historia se refieren a conflictos bélicos, sus fases o su situación —mención especial merece el contenido que se refiere a "la globalización económica, las relaciones interregionales en el mundo, los focos de conflicto y los avances tecnológicos" (ORDEN EDU/362/2015, p. 32152) en la que parece que se pueda establecer una relación entre todos esos elementos y acercarse de ese modo a la EDCG-; en Lengua Castellana y Literatura se utiliza el teatro "como modo de aprendizaje y expresión oral, de conocimiento de conductas y conflictos (...)" (ORDEN EDU/362/2015, p. 32165 ); en Primera Lengua extranjera se hace referencia a que el uso de lenguas extranjeras facilitará la "simbiosis entre culturas que facilitará la resolución de conflictos de manera satisfactoria” (ORDEN EDU/362/2015, p. 32232).

Por último, es en Valores Éticos donde más referencia se hace al conflicto, a su resolución y a la manera de entenderlo más cercana a la EDCG. Aparece tanto en la presentación de la asignatura como en los contenidos, los criterios de evaluación y los estándares de aprendizaje evaluables. En esta asignatura se afirma que se deben reconocer los "derechos humanos como referencia universal para superar los conflictos, defender la igualdad, el pluralismo político y la justicia social" (ORDEN EDU/362/2015, p. 32341). Además, por ejemplo, también se acerca a la EDCG cuando se habla de la globalización, "la desigualdad, la interdependencia, la internacionalización de los conflictos armados, la imposición de modelos culturales determinados por intereses económicos (...)” (ORDEN EDU/362/2015, p. 32358).

\section{Conclusiones y discusión}

El análisis realizado de la presencia de las desigualdades y la resolución pacífica de conflictos permite extraer dos conclusiones:

1. Las desigualdades solo aparecen tratadas en tres asignaturas de ESO y, en algunos casos, ni siquiera en los contenidos de estas. De esta forma, no se hace hincapié en las desigualdades 
existentes entre países ni en las causas de estas. Además, el hecho de que las desigualdades sean abarcadas solo en las asignaturas de Economía, Geografía e Historia y Valores éticos nos desvela que la presencia que tiene en la normativa tiende a hacerse en materias optativas que el alumnado puede o no cursar.

2. La resolución pacífica de conflictos está presente en la normativa como forma de solucionar los problemas para garantizar la convivencia. Es decir, se trata de una noción que se suele referir especialmente a los conflictos personales o escolares, y poco a los conflictos sociales a los que también debería hacerse alusión para la formación de ciudadanos globales. De hecho, únicamente en una, la asignatura optativa de Valores éticos, se hace referencia a la resolución del conflicto relacionándolo con los derechos humanos y la justicia social.

Con estos resultados, se puede señalar que el tratamiento de las desigualdades debe cambiar y adquirir mayor peso en el currículo para comprender las causas de las desigualdades existentes no solo a nivel local, sino entre países. Autores como Belmonte y Guillamón (2008) o Pino, Galeano y Correa (2019) remarcan que la escuela debe coeducar e impulsar la igualdad de género entre las cuestiones a tratar con su alumnado. Sin embargo, se debe abarcar la diversidad en todas sus formas para formar ciudadanos globales que piensen críticamente y apuesten por el cumplimiento de los derechos humanos (Nava-Preciado y Méndez-Huerta, 2019; Rodríguez, 2019).

Adicionalmente, la resolución pacífica de conflictos, como se ha podido apreciar, tiene presencia en la normativa, pero debería focalizarse también en los conflictos que afectan a la sociedad para adquirir una perspectiva de los problemas que afectan a la ciudadanía de otras partes del mundo. En este sentido, se debe tener presente que "el marco educativo es el entorno idóneo para aprender las estrategias de solución pacífica de conflictos tanto intrapersonales como interpersonales" (Moliner y Martí, 2002, p. 2). Por ello, "sería imprescindible apostar por una escuela democrática, pacífica e inclusiva con la implicación de toda la comunidad” (Alguacil et al., 2019, p. 81).

En síntesis, la normativa que regula el currículo de ESO en Castilla y León (España) si trata las desigualdades y la resolución pacífica de conflictos, pero no de una manera idónea puesto que estas cuestiones deben adquirir una mayor presencia en las asignaturas e incluir cuestiones que afectan a la sociedad global.

\section{Referencias}

Alguacil, M., Boqué, M. C., y Alcalde, M. D. R. (2019). Educar para la Paz y en la Paz: Elementos a Considerar en la Escuela. Revista Internacional de Educación para la Justicia Social, 8(2), 65-87. DOI: https://doi.org/10.15366/riejs2019.8.2.004

Andreotti, V. (2014). Critical and transnational literacies in international development and global citizenship education. Journal of education, 2(3), 32-50.

Bermeo-Yaffar, F., Hernández-Mosqueda, J. S., y Tobón-Tobón, S. (2016). Análisis documental de la V heurística mediante la cartografía conceptual. Ra Ximhai, 12(6), 103-121. DOI: https://doi.org/10.35197/rx.12.01.e3.2016.05.fb

Bourn, D. (2015). The Theory and Practice of Development Education: A pedagogy for global social justice. Nueva York: Routledge.

\section{Se puede}

señalar que el

tratamiento de las

desigualdades

debe cambiar y

adquinir mayor

peso en el currículo

para comprender

las causas de las

desigualdades

existentes no solo

a nivel local, sino

entre países 
Caride, J. A. (2017). Educación social, derechos humanos y sostenibilidad en el desarrollo comunitario. Teoría de la educación, 29(1), 245-272. DOI: https://doi.org/10.14201/teoredu291245272

Lapponi, S. F. (2013). Resolución de conflictos en la escuela: una herramienta para la cultura de paz y la convivencia. Contextos Educativos. Revista de Educación, (3), 91-106.

Mayoral, S. (2011). Los enfoques de la educación para el desarrollo en España. Acciones e investigaciones sociales, (30), 43-75. DOI: https://doi.org/10.26754/ojs_ais/ais.201130604

Molina, M. P. (1989). Introducción al análisis documental y sus niveles: el análisis de contenido. Boletín de la ANABAD, 39(2), 323-342.

Moliner, O., y Martí, M. (2002). Estrategias didácticas para la solución cooperativa de conflictos y toma de decisiones consensuadas: mejorar la convivencia en el aula. Revista electrónica interuniversitaria de formación del profesorado, 5(3), 11.

Murga-Menoyo, M. (2018). La Formación de la Ciudadanía en el Marco de la Agenda 2030 y la Justicia Ambiental. Revista Internacional de Educación para la Justicia Social, 7(1), 37-52. DOI: https://doi.org/10.15366/riejs2018.7.1.002

Nava-Preciado, J. M., y Méndez-Huerta, M. (2019). Adolescentes y derechos humanos: una aproximación a su ejercicio efectivo en la escuela. Revista Latinoamericana de Ciencias Sociales, Niñez y Juventud, 17(1), 285-299.

OECD. (2018). Marco de Competencia Global Estudio PISA. Preparar a nuestros jóvenes para un mundo inclusivo y sostenible. Madrid: Ministerio de Educación, Cultura y Deporte.

ORDEN EDU/362/2015, de 4 de mayo, por la que se establece el currículo y se regula la implantación, evaluación y desarrollo de la educación secundaria obligatoria en la Comunidad de Castilla y León. Recuperado de https:/www.educa.jcyl.es/es/resumenbocyl/ orden-edu-362-2015-4-mayo-establece-curriculo-regula-implan.ficheros/549394-BOCYL-D-08052015-4.pdf

Organización de Naciones Unidas. (2015). Transformar nuestro mundo: la Agenda 2030 para el Desarrollo Sostenible. Nueva York: ONU.

Ortega, M. L., Cordón-Pedregosa, M. R., y Sianes, A. (2013). La Educación para el desarrollo en el espacio universitario: de la formación en contenidos a la formación integral. Revista Española del Tercer Sector, (25), 53-78.

Ortega, R. (2006). La convivencia escolar y los retos de la educación en el siglo XXI. En E. Iguaz de Miguel (ed.), Foro para la convivencia en los centros educativos (pp. 17-50). Granada: Consejo Escolar de Andalucía.

Pashby, K. (2015). Conflations, possibilities, and foreclosures: Global citizenship education in a multicultural context. Curriculum Inquiry, 45(4), 345-366. DOI: https://doi.org/10.10 80/03626784.2015.1064304

Pashby, K., Costa, M., Stein, S., \& Andreotti, V. (2020). A meta-review of typologies of global citizenship education. Comparative Education, 56(2), 144-164. DOI: https://doi.org/10. 1080/03050068.2020.1723352

Pino, A., Galeano, E. B., y Correa, R. (2019). Hacia una escuela inclusiva: perspectiva de género y literatura. Polyphōnía: Revista de Educación Inclusiva, 3(1), 66-76. 
Prada, J. de, y López, J. (2008). La mediación como estrategia de resolución de conflictos en el ámbito escolar. Documentación social, 148, 99-116.

Rebollo, M. Á. (2006). Género e interculturalidad: educar para la igualdad. Madrid: Editorial La Muralla.

Rodríguez, Y. G. (2019). Diversidades culturales desde una concepción plural en el aula de Educación Primaria y Secundaria. Revista Prisma Social, (25), 66-83.

Salazar-Gomez, E., y Tobon, S. (2018). Análisis documental del proceso de formación docente acorde con la sociedad del conocimiento. Espacios, 39(45), 17.

Sanahuja, J. A. (2015). La Agenda 2030 y los Objetivos de Desarrollo Sostenible: hacia una ética universalista del desarrollo global. Razón y fe, 272(1405), 367-381.

Santamaría-Cárdaba, N. (2018). Educando para el desarrollo y la ciudadanía global a través de la psicología positiva. REIDOCREA, 7, 98-109.

Soriano, A. (2009). Violencia y conflicto. La escuela como espacio de paz. Profesorado, Revista de Currículum y Formación del Profesorado, 13(1), 321-334.

Valles, M. S. (1999). Técnicas cualitativas de investigación social. Reflexión metodológica y práctica profesional. Madrid: Síntesis.

Vila, E. (2009). Educar para la tolerancia, educar para la convivencia. Espiral. Cuadernos del profesorado, 2(4), 43-47. DOI: http://dx.doi.org/10.251 15/ecp.v2i4.895 